05

\title{
Численное моделирование процесса получения мультикремния методом направленной кристаллизации
}

\author{
(C) С.А. Смирнов, В.В. Калаев \\ ООО „Софт-Импакт“, \\ 194044 Санкт-Петербург, Россия \\ e-mail: sergey.smirnov@str-soft.com
}

Поступило в Редакцию 22 апреля 2019 г.

В окончательной редакции 25 декабря 2019 г.

Принято к публикации 19 января 2020 г.

Рассмотрен метод численного моделирования процесса направленной кристаллизации мультикремния в тигле квадратного сечения. Дано обоснование применения в расчетах $2 D$-осесимметричной геометрии, построенной по вертикальному сечению печи. Математическая модель описывает гидродинамику расплава, течение газа, глобальный теплообмен, тепловые напряжения и эволюцию плотности дислокаций в растущем кристалле. Определена чувствительность напряжений и плотности дислокаций к параметрам модели Александера-Хаазена.

Ключевые слова: кристалл, гидродинамика, тепловые напряжения, дислокации.

DOI: 10.21883/JTF.2020.07.49440.169-19

\section{Введение}

Мультикремний - это кристаллический материл с блочной структурой, состоящий из одинаково ориентированных достаточно крупных монокристаллических зерен. Для получения оптоэлектронных подложек кристаллы мультикремния в основном выращивают из расплава методом направленной кристаллизации (Directional Solidification System - DSS). Их качество зависит от типа и количества примесных атомов, плотности дислокаций, размеров и ориентации граней монокристаллических зерен. Для производства кристаллов необходима организация длительных ресурсно-затратных процессов. Численное моделирование позволяет оптимизировать режимы работы ростовых печей и выполнить анализ структурных характеристик кристаллов. Например, в [1] было показало, что понижение потребляемой мощности нагревателей печи достигается при относительно небольшом изменении конструкции тепловой изоляции. В [2] найдены условия для обеспечения плоского ростового интерфейса (межфазной границы кристалл/расплав) с минимальными тепловыми напряжениями в кристалле. В [3] для ростовой стадии процесса определен алгоритм движения боковой теплоизоляции при высокой скорости роста кристалла со слегка выпуклым интерфейсом и с пониженным уровнем напряжений. В [4] для стадии охлаждения выращенного кристалла исследовано влияние скорости движения боковой теплоизоляции на остаточные напряжения и плотность дислокаций. В этих работах были рассмотрены промышленные печи с тиглями, имеющими в горизонтальном сечении форму квадрата. Для такой формы тигля детальное описание нестационарных процессов возможно только в $3 D$-приближении при значительных затратах машинного времени и, следовательно, при сниженной эффективности численного сопровождения технологических работ. Поэтому расчеты [1-4] выполнялись в $2 D$-осесимметричной геометрии, построенной для вертикальной плоскости симметрии печи параллельной грани тигля. Несмотря на данное упрощение, в [2-4] реализация в производстве рекомендаций расчетов позволила увеличить эффективность солнечных элементов. В упомянутых выше работах использовался специализированный код CGSim [5].

B настоящей работе путем сравнения полей температур и напряжений $3 D$ и $2 D$ тестовых расчетов дано обоснование использования модельного $2 D$-осесимметричного приближения для анализа процессов в тигле квадратного сечения. Также проведено моделирование промышленного процесса получения кристалла в печи, конструктивно аналогичной [1-4], с использованием кода CGSim [5].

Математическая модель настоящей работы описывает гидродинамику расплава, течение газа в объеме печи, теплопроводный и лучистый теплообмен, рост кристалла с самосогласованным расчетом формы ростового интерфейса, тепловые напряжения в кристалле и эволюцию плотности дислокаций. Уделено внимание граничным условиям по напряжениям на границах кристалла с тиглем, сильное влияние которых на характеристики кристалла для печи лабораторного масштаба показано в [6,7]. При расчете плотности дислокаций по модели Александера-Хаазена (AX) [8] нами использованы функциональные зависимости некоторых параметров Si-кристаллов, определенные по экспериментальным данным [9]. Данные зависимости целесообразно использовать при численной оптимизации процессов получения кристаллов, в частности, при поиске алгоритмов постростового охлаждения, позволяющих уменьшить плотность дислокаций без увеличения продолжительности полного процесса [10,11]. 


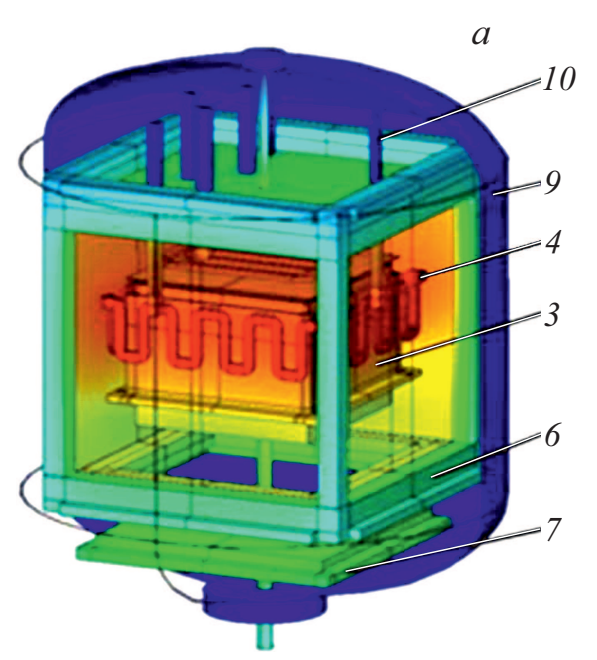

$c$

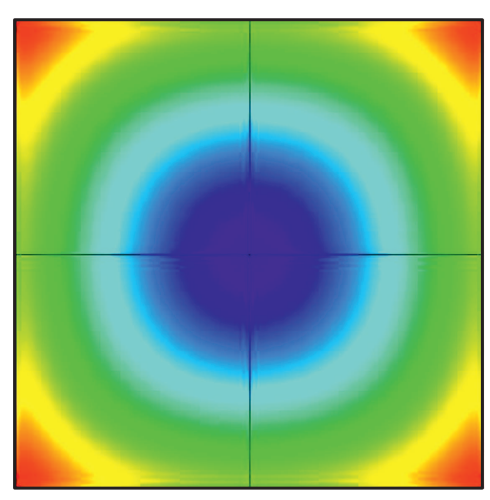

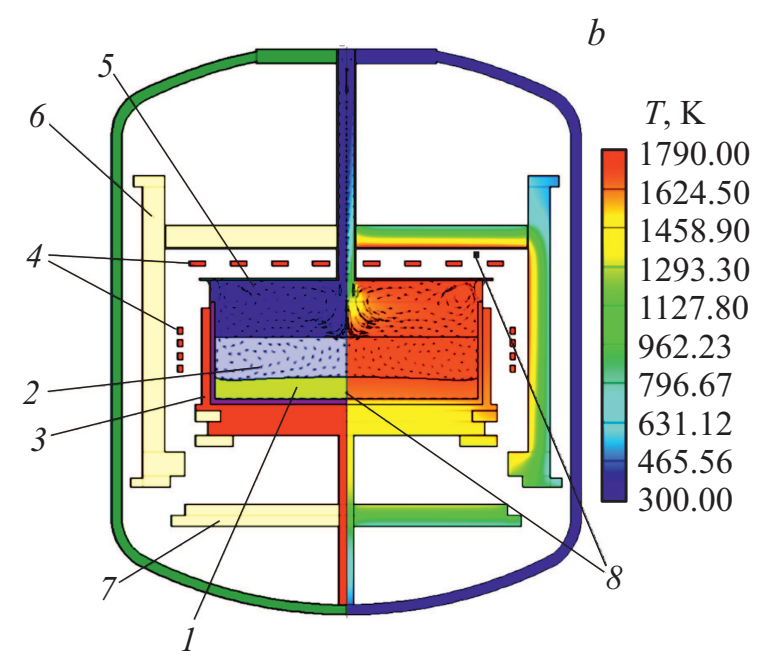

$d$

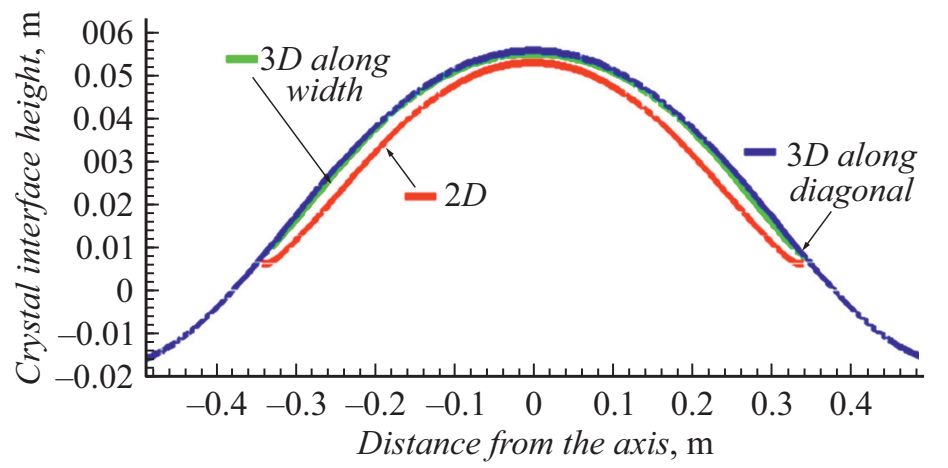

Рис. 1. Расчетные геометрии печи и результаты тепловых тестовых расчетов. $a$ - $3 D$-детальная геометрия; $b-$ $2 D$-осесимметричная геометрия; $c$ - распределение температуры в горизонтальном сечении $3 D$-загрузки; $d-$ формы изотерм $1685 \mathrm{~K}$ в $2 D$ - и $3 D$-расчетах. 1 - кристалл; 2 - расплав; 3 - тигель; 4 - нагреватели; 5 - газовый объем над расплавом; 6 - боковая подвижная теплоизоляция; 7 - нижний блок теплоизоляции; 8 - места расположения измерительных спаев термопар; 9 - корпус; 10 - токовод.

\section{Геометрия расчетной области и результаты тестовых расчетов}

Общий $3 D$-вид печи приведен на рис. $1, a$. Во внешнем водоохлаждаемом корпусе расположены тигель, резистивные нагреватели, теплоизоляция. К поверхности расплава по вертикальному каналу подается чистый $\mathrm{N}_{2}$ или $\mathrm{Ar}$ для выноса из внутреннего объема печи примесей, загрязняющих кристалл. Осесимметричный модельный $2 D$-аналог этой конструкции показан на рис. $1, b$, он соответствует [1]. Преимущество $2 D$-приближения заключается в простоте подготовки модельной геометрии и увеличении скорости расчетов. Как было показано выше, его практическая ценность подтверждена в [2-4], в настоящей работе нами определена пространственная область корреляции $2 D$ и $3 D$ тестовых расчетов.

Выполнено два теста. Первый - с использованием модели глобального теплообмена при неподвижном расплаве с детальной проработкой геометрии росто- вого пространства печи (рис. 1,a). Соответствующее распределение температуры в горизонтальном сечении, проходящем через середину высоты загрузки $3 D$-тигля, показано на рис. 1,c. Видно, что в большей части этого сечения температурное поле осесимметрично, в результате в $3 D$ - и $2 D$-геометриях хорошо совпадают изотермы $T_{\mathrm{TSol} / \mathrm{Liq}}=1685 \mathrm{~K}$ (рис. $1, d$ ). Отсюда можно сделать вывод, что осесимметричный $2 D$-расчет может использоваться для получения данных о форме ростового интерфейса в большей части объема реального $3 D$-тигля с дном квадратного сечения, а именно в области вертикального цилиндра, вписанного в $3 D$-тигель с квадратным дном.

Во втором тесте сравнивались напряжения VMS (Von Mises Stress) для цилиндра и куба с линейным изменением температуры по высоте боковых границ (рис. 2,a,b). Длина ребер куба, диаметр и высота цилиндра равны $100 \mathrm{~mm}$. На рис. 2, $a, b$ иниями отмечены вертикальные сечения, развернутые на рис. 2, $c-e$. В том 

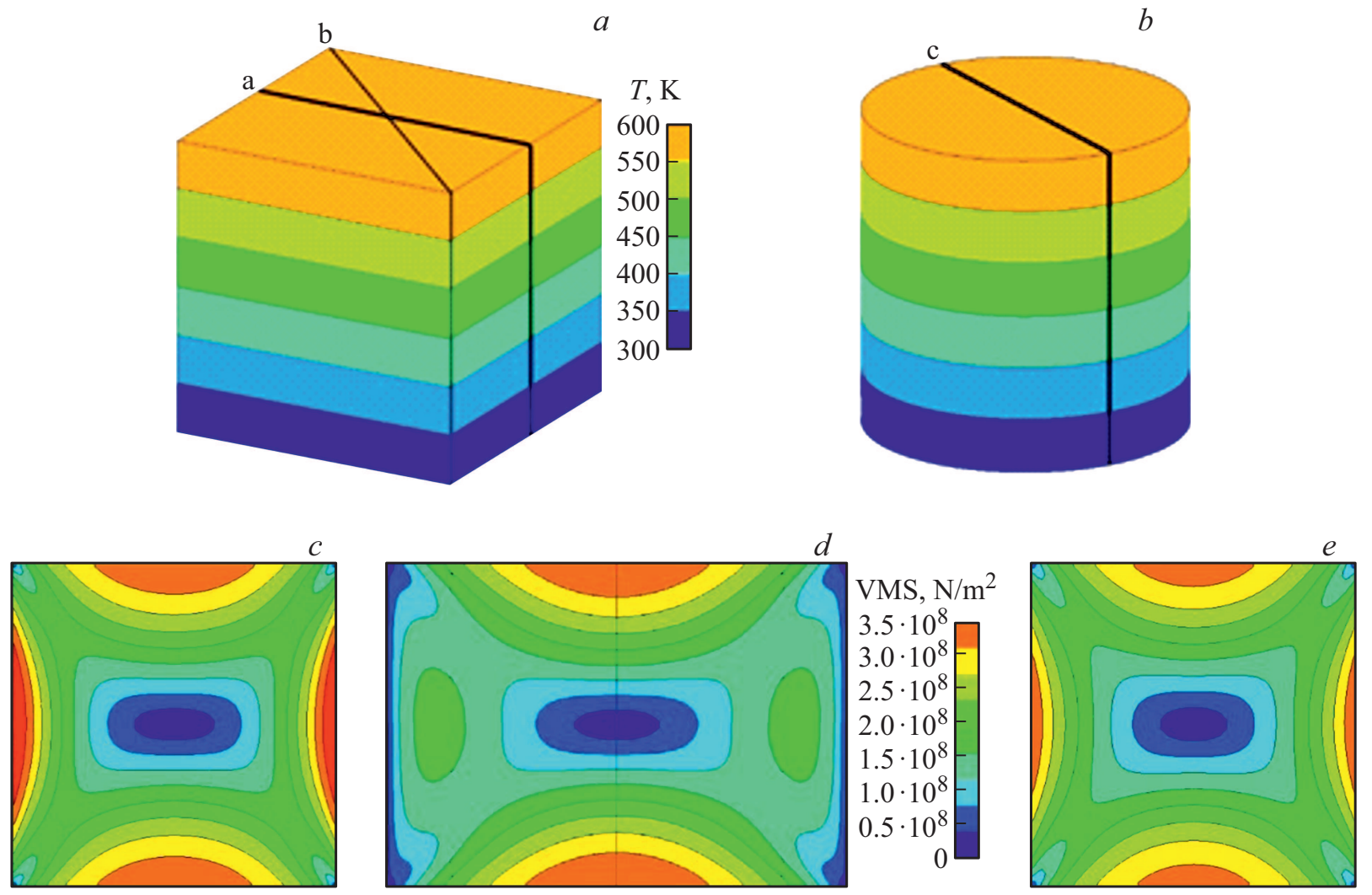

Рис. 2. Тестовые геометрии и распределения VMS в вертикальных сечениях куба $(a)$ и цилиндра $(b)$, проведенных через ось: $c$ - параллельно грани куба; $d$ - по диагонали куба; $e-$ по оси цилиндра.

числе широтное сечение куба, проходящее через его ось параллельно боковой грани (рис. 2,c); диагональное сечение куба (рис. 2,d); осевое сечение цилиндра (рис. 2,e). Видно, что в широтном сечении куба (рис. 2,c) и в осевом цилиндра (рис. 2,e) распределения VMS удовлетворительно согласуются друг с другом. В диагональном сечении куба (рис. 2, $d$ ), а именно в его периферийных частях с сильным влиянием углов, наблюдаются заметные отличия от цилиндрического распределения (рис. 2,e).

На рис. 3 приведены распределения VMS по верхней грани куба (рис. $3, a)$ и по горизонтальному сечению, проходящему через середину его высоты (рис. $3, b$ ). Из pис. $3, a$ следует, что на значительной части площади верхней грани куба распределение VMS близко к осесимметричному. В срединном горизонтальном сечении куба цилиндричность VMS можно принять лишь приближенно для приосевой области с радиусом до четверти ширины $3 D$-тигля.

На рис. 4 приведены графики распределения VMS вдоль линий пересечения вертикальных сечений $(a-c$ на рис. 2), с верхними гранями и со срединными горизонтальными сечениями куба и цилиндра. Данные графики подтверждают корреляцию распределений VMS вертикального осевого сечения цилиндра и широтного сечения куба. Заметное рассогласование наблюдается для вертикального сечения цилиндра и диагонального куба.

По результатам этих тестов можно сделать вывод о том, что при использовании осесимметричной $2 D$-геометрии, построенной по вертикальному широтному сечению тигля, расчетные распределения температуры и VMS будут удовлетворительно коррелировать с параметрами полного $3 D$-расчета только в области условного вертикального цилиндра, вписанного в $3 D$-тигель. Как следствие предыдущего, при использовании $2 D$-приближения можно рассчитывать на получение правдоподобной формы интерфейса в широтном сечении $3 D$-тигля.

Отметим, что если в $2 D$-геометрии ростовой печи внутренний радиус тигля задать равным полуширине $3 D$ реального, т.е. положить $r=a$ (здесь $r-$ радиус модельного $2 D$ - тигля, а $a-$ полуширина реального $3 D$-тигля), то при одной и той же высоте кристалла в $2 D$-расчете его масса будет в $\pi / 4$ меньше реальной, так как площади оснований цилиндра и куба будут соответственно равны $S_{2 D}=\pi a^{2}$ и $S_{3 D}=4 a^{2}$. При фиксированных величинах теплопроводности и коэффициентах черноты поверхностей блоков печи это различие объемов ухудшит согласование расчетных и экспериментальных мощностей нагревателей. Поэтому, когда необходимо повысить точность расчета потребляемой мощности, в $2 D$-геометрии радиусы тигля, боковых нагревателей 

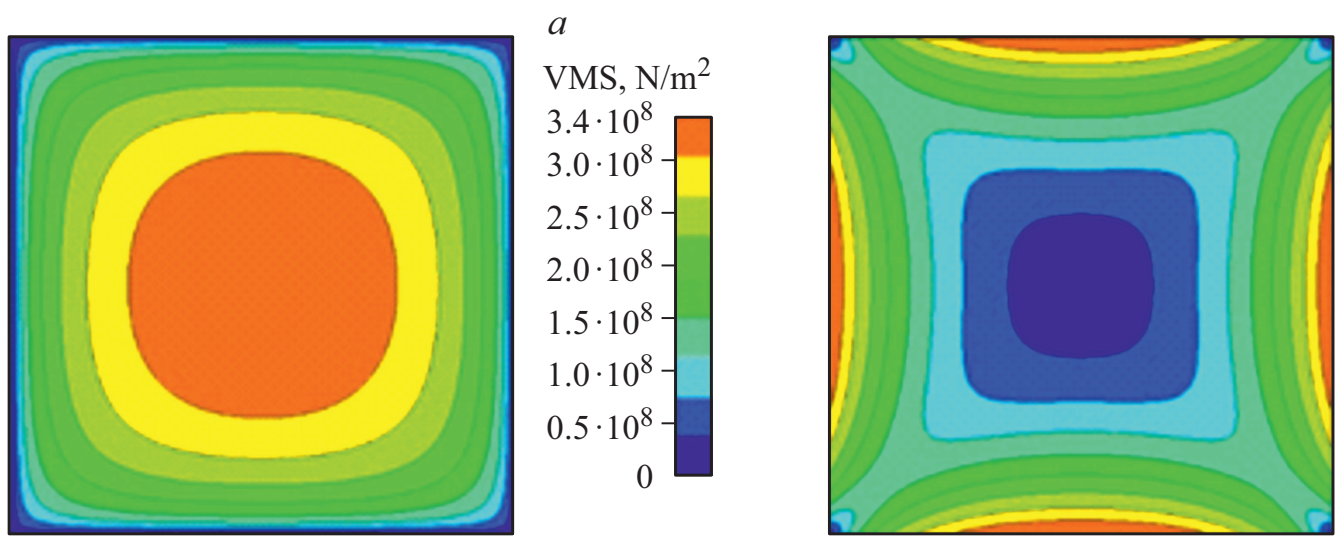

$b$

$\mathrm{VMS}, \mathrm{N} / \mathrm{m}^{2}$

$4.2 \cdot 10^{8}$

$4.0 \cdot 10^{8}$

$3.5 \cdot 10^{8}$

$3.0 \cdot 10^{8}$

$2.5 \cdot 10^{8}$

$2.0 \cdot 10^{8}$

$1.5 \cdot 10^{8}$

$1.0 \cdot 10^{8}$

$0.5 \cdot 10^{8}$

0

Рис. 3. Распределения VMS в горизонтальных сечениях куба: $a-$ на верхней грани; $b-$ посередине высоты.
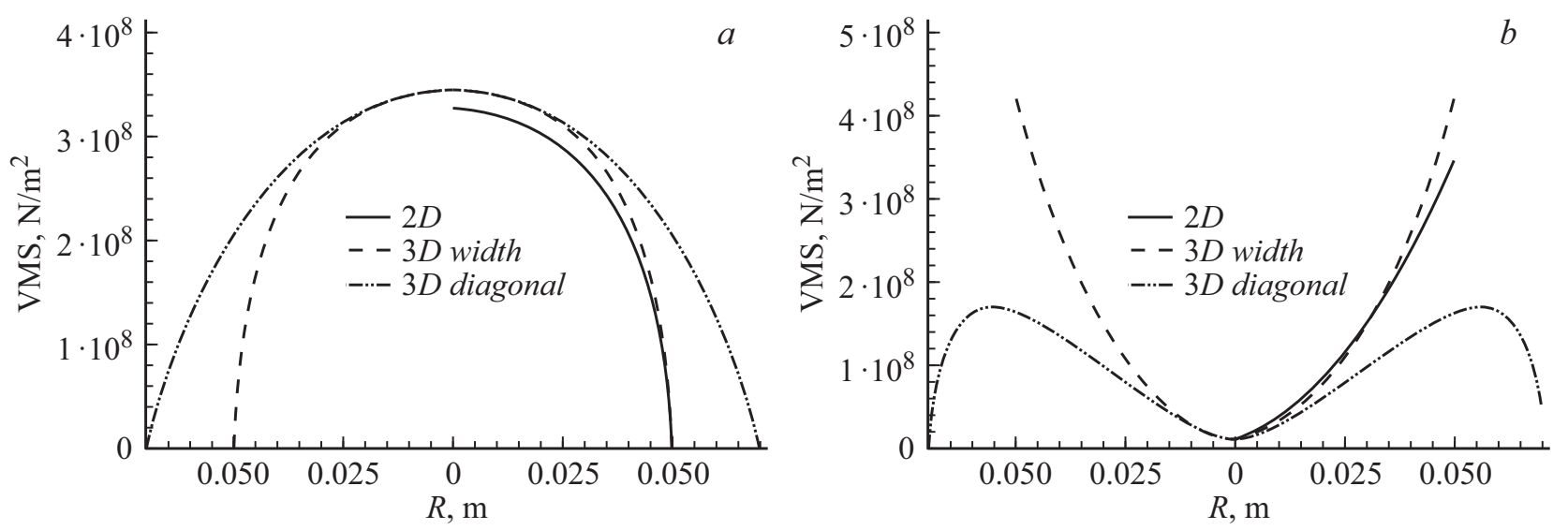

Рис. 4. Распределения VMS вдоль линий в $2 D$ - и $3 D$-расчетах: $a-$ верхняя грань; $b$ - горизонтальное сечение посередине высоты.

и теплоизоляции масштабируются с коэффициентом $(4 / \pi)^{0.5}$, т. е. для модельного тигля с осевой симметрией надо использовать радиус $r_{\mathrm{eff}}=(4 / \pi)^{0.5} a$.

\section{Математическая модель}

Математическая модель работы описывает тепловой баланс всех элементов печи. В ней также учтено турбулентное движение расплава и газа. В растущем кристалле исследуются динамика тепловых напряжений и плотности дислокаций. Форма межфазной границы расплав/кристалл для каждого расчетного момента времени определяется по балансу локальных тепловых потоков на данном интерфейсе. Самосогласованные динамические расчеты полного технологического процесса выполнялись кодом CGSim [5]. При расчете тепловых напряжений учитывалась их релаксация при генерации дислокаций, для этого использована модель АХ. Параметры турбулентности свободно конвективного течения расплава и потока газа вычислялись по модели Вольфштейна [12]. Наличие развитой турбулентности в подобном DSS-процессе, когда температура расплава увеличивается вверх и, казалось бы, нет условий для заметной свободной конвекции, было установлено в $3 D$-нестационарных расчетах [13].

Гидродинамическая и тепловая части модели описаны в ряде работ, посвященных процессам роста кристаллов из расплава, а также в описании [5]. Здесь остановимся на модели дислокаций $\mathrm{AX}[8]$. В ней результирующая деформация $\varepsilon_{i j}$ определяется суперпозицией упругой, тепловой и пластичной $\varepsilon_{i j}^{e}, \varepsilon_{i j}^{T}$ и $\varepsilon_{i j}^{c}$ соответственно:

$$
\varepsilon_{i j}=\varepsilon_{i j}^{e}+\varepsilon_{i j}^{T}+\varepsilon_{i j}^{c} .
$$

Тепловая деформация вызывается изотропным тепловым расширением

$$
\varepsilon_{i j}^{T}=\frac{\delta_{i j}}{3}\left[1-\frac{\rho_{\mathrm{Si}}(T)}{\rho_{\mathrm{Si}}\left(T_{r e f}\right)}\right] .
$$

Здесь $\rho_{\mathrm{Si}}-$ плотность кристалла $\left(\rho_{\mathrm{Si}}=2302.3-0.03084 \cdot T\left[\mathrm{~kg} / \mathrm{m}^{3}\right]\right), \quad T-$ локальная температура, $T_{r e f}$ - температура недеформированного материала, для кремния принято $T_{r e f}=1685 \mathrm{~K}$ (температура плавления). Результирующая деформация $\varepsilon_{i j}$ 
связана с компонентами сдвига $u_{i}$ и $u_{j}$ уравнением

$$
\varepsilon_{i j}=\frac{1}{2}\left(\frac{\partial u_{i}}{\partial x_{j}}+\frac{\partial u_{j}}{\partial x_{i}}\right) .
$$

Скорость изменения пластичной деформации и плотности движущихся дислокаций $N_{m}$ записывается уравнениями

$$
\begin{gathered}
\varepsilon_{i j}=\frac{1}{2} b N_{m} \frac{1}{\sqrt{J_{2}}} S_{i j} V, \\
V=k_{0}\left(\tau_{\mathrm{eff}}\right)^{p} \exp \left(-\frac{Q}{k T}\right), \\
\frac{d N_{m}}{d t}=K k_{0}\left(\tau_{\mathrm{eff}}\right)^{p+\lambda} \exp \left(-\frac{Q}{k T}\right) N_{m}-\frac{N_{m} V}{L}, \\
\tau_{\mathrm{eff}}=\sqrt{J_{2}}-D \sqrt{N_{m}}, \\
D=R \frac{E b}{4 \pi\left(1-v^{2}\right)} \\
J_{2}=\frac{1}{2} \sum_{i, j} S_{i j}^{2}, \\
S_{i j}=\sigma_{i j}-\delta_{i j} \frac{1}{3} \sum_{k} \sigma_{k k},
\end{gathered}
$$

где $b$ - модуль вектора Бюргерса; $\tau_{\mathrm{eff}}-$ эффективное напряжение; $Q$ - потенциал Пайерлса; $k-$ постоянная Больцмана; $S_{i j}$ - девиатор напряжений; $J_{2}-$ второй инвариант девиатора напряжений; $D$ и $R-$ коэффициент и относительный коэффициент деформационного упрочнения соответственно; $E$ - модуль Юнга; $v$ - коэффициент Пуассона; $k_{0}, K, p, \lambda$ - постоянные материала в модели $\mathrm{AX} ; \sigma_{i j}$ - компоненты тензора напряжений, $N_{m}$ - плотность подвижных дислокаций, $L-$ средний линейный масштаб грани монокристаллического зерна, $V$ - средняя скорость движения дислокаций. В случае, когда $\sqrt{J_{2}}-D \sqrt{N_{m}}<0$, величина $\tau_{\text {eff полагается равной }}$ нулю, т.е. скорость пластичной деформации и скорость генерации дислокаций - нули. Упругая деформация $\varepsilon_{i j}^{e}$ и полное напряжение $\sigma_{i j}$ выражаются через уравнение сохранения момента и закон Гука:

$$
\begin{gathered}
\sum_{j} \frac{\partial \sigma_{i j}}{\partial x_{j}}=0, \\
\sigma_{i j}=\sum_{j} c_{i j} \varepsilon_{i j}^{e},
\end{gathered}
$$

где $c_{i j}$ - упругие постоянные.

Для $\mathrm{Si}$-кристалла в модели $\mathrm{AX}$ за базовые, как правило, берут величины $p=1.1, Q=2.2 \mathrm{eV}$, $\lambda=1, R=0.8165, b=3.8 \cdot 10^{-10} \mathrm{~m}, K=3.1 \cdot 10^{-4} \mathrm{~m} / \mathrm{N}$, $k_{0}=8.58 \cdot 10^{-4} \mathrm{~m}^{2 p+1} / \mathrm{N}^{\mathrm{p}} / \mathrm{s}$, например, [14]. Фиксированные значения $p=1.1, Q=2.2 \mathrm{eV}$ были предложены в [15] на основании экспериментальных данных для температуры, близкой к температуре плавления. Но в рассматриваемом здесь полном процессе получения кристаллов активная генерация дислокаций происходит на этапе охлаждения, где параметры $p$ и $Q$ могут иметь другие значения. Поэтому представляет интерес уточнение величин $p$ и $Q$, анализ их влияния на результаты моделирования.

Мы провели расчеты как при фиксированных параметрах $p=1.1, Q=2.2 \mathrm{eV}$, так и при функциональных зависимостях $p(T)$ и $Q\left(\tau_{\text {eff }}\right)$, полученных на основе экспериментальных данных [9]. Величина $p(T)$ в [9] при изменении температуры $T$ от 773 до $1073 \mathrm{~K}$ уменьшалась от 1.43 до 1.30. Эта закономерность $p(T)$ напрямую была применена в расчетах, а для $T<773 \mathrm{~K}$ и $T>1073 \mathrm{~K}$ задавалась линейная экстраполяция $p(T)$. График $Q\left(\tau_{\mathrm{eff}}\right)$, построенный по данным [9], приведен на рис. 5.

В рассматриваемой конструкции промышленной печи кристалл выращивают в тигле, внутренняя часть которого изготовлена из кварца, а внешняя из графита. Для самосогласованности расчетов термоупругих напряжений необходимо учитывать эту структуру тигля и условия его крепления в печи. В настоящей работе мы вычисляем напряжения только в кристалле, что требует постановки граничных условий по напряжениям на нижней и на боковых поверхностях кристалла, касающихся внутренней кварцевой части тигля. В силу пластичности кварца, проявляющейся при высоких температурах, эти граничные условия находятся между типами „свободной“ границы, смещение которой не ограничено, и „фиксированной“ границы с нулевым перемещением. Переход от первого типа граничного условия ко второму меняет результат расчета напряжений примерно на порядок, при этом определение точного промежуточного условия достаточно сложно. С целью упрощения мы приняли во внимание, что при высоких температурах кварц является пластичным буфером между кристаллом и внешним графитовым тиглем. Для моделирования этого эффекта в относительно тонком слое кристалла толщиной $30 \mathrm{~mm}$ по границе с тиглем задали линейное изменение величины модуля Юнга от $E_{\mathrm{Si}}=1.66 \cdot 10^{11} \mathrm{~Pa}$, соответствующего $\mathrm{Si}$-кристаллу, до значения кварца. С наружной стороны этого буферного слоя ставилось условие фиксированной границы. На верхней поверхности кристалла выполнялось условие „свободная граница“.

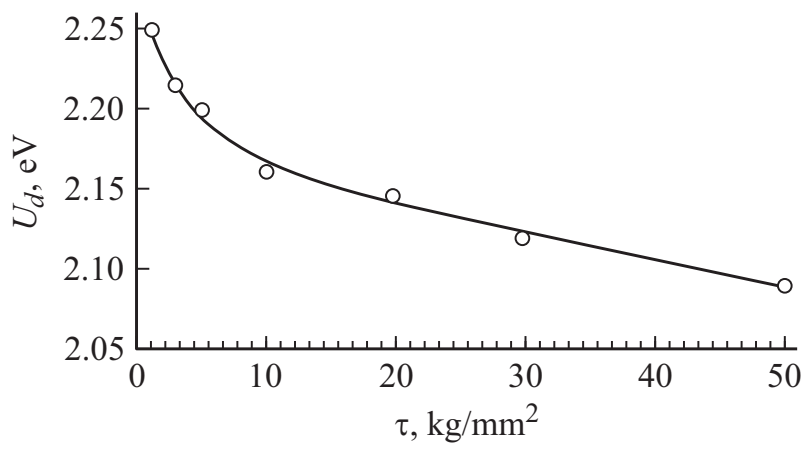

Рис. 5. Зависимость энергии активации от напряжения, потенциал Пайерлса, рисунок из [9]. 


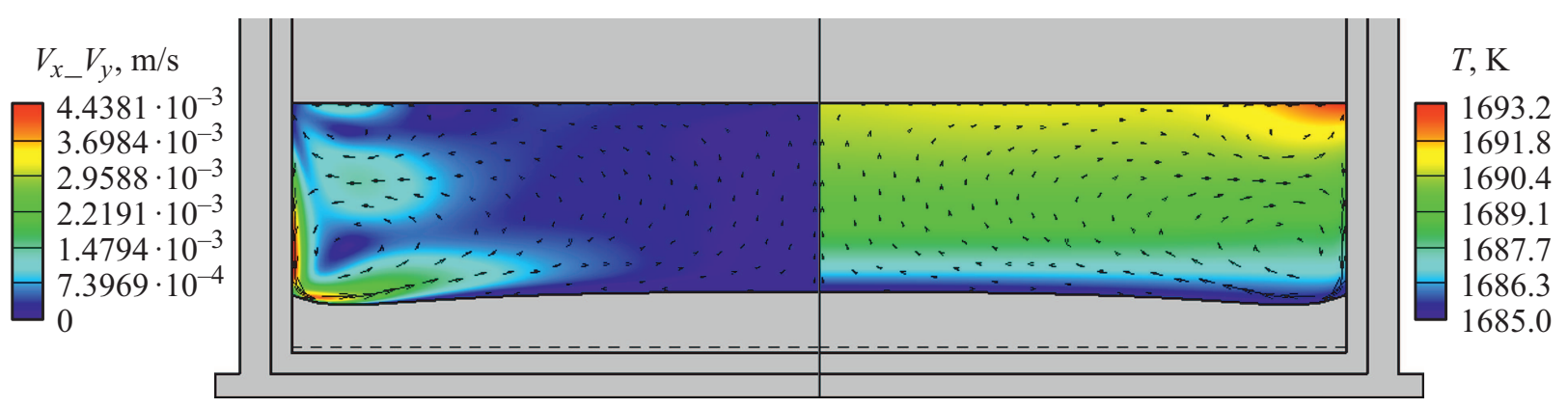

Рис. 6. Распределение скорости и температуры расплава, $t=7 \mathrm{~h}$.
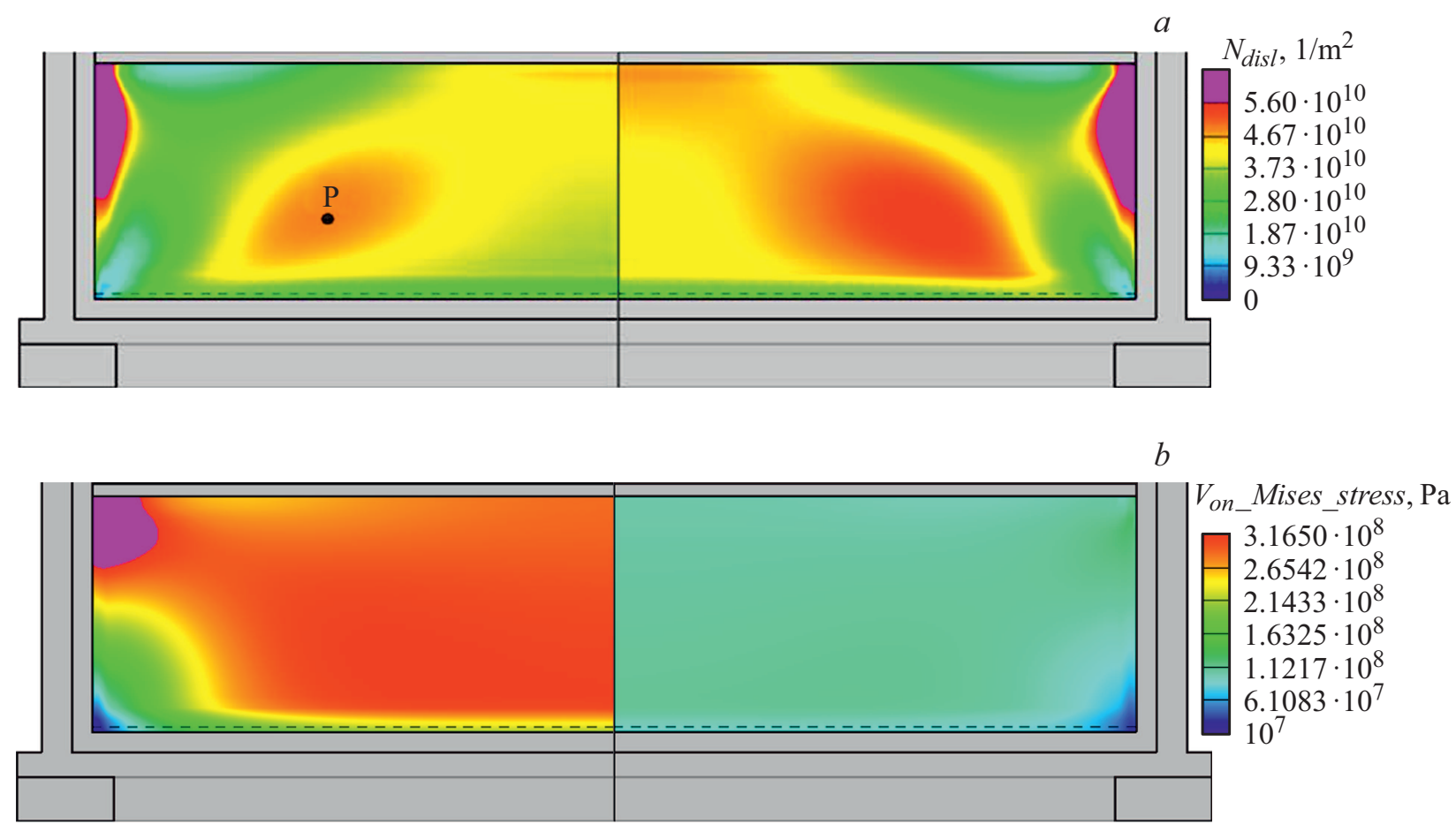

Рис. 7. Распределение и VMS: слева $-Q=2.2 \mathrm{eV}, p=1.1$, справа $-Q\left(\tau_{\text {eff }}\right)$ и $p(T), t=50 \mathrm{~h}$.

\section{Результаты моделирования кристаллизации}

В производстве мультикремния производственные стадии - плавка загрузки, начальная кристаллизация, основной процесс роста, пост-ростовая закалка и охлаждение кристалла - обеспечиваются изменением мощностей нагревателей и регулировкой тепловых потоков от дна тигля к внешним стенкам печи. Мощность радиационных потоков от нижней части тигля к охлаждаемому корпусу зависит от площади зазора, создаваемого между блоками теплоизоляции. Она меняется по заданному алгоритму смещением боковой теплоизоляции относительно нижней. В коде CGSim обеспечена возможность моделирования такого смещения теплоизоляции при одновременной перестройке расчетной сетки и пересчете коэффициентов радиационного теплообмена („view factors“'). Для изменения мощности нагревателей по заранее заданным алгоритмам используется численный аналог пропорционально-интегральнодифференцирующего (ПИД) алгоритма автоматической системы управления печи. ПИД-алгоритм регулирует мощность нагревателей по заданным временным зависимостям температур термопар, а термопары - это точки мониторинга температуры в твердых блоках печи или в специально построенных блоках небольшого размера, размещенных в газовом объеме.

Здесь нами рассмотрен процесс продолжительностью $50 \mathrm{~h}$ при росте кристалла в течение $28 \mathrm{~h} \mathrm{c}$ последующим отжигом и охлаждением за $22 \mathrm{~h}$. Эффективный диаметр кристалла $r_{\text {eff }}=1075 \mathrm{~mm}$, загрузка кремния $600 \mathrm{~kg}$. Рассмотрены два набора параметров $Q$ и $p$, в первом случае $Q=2.2 \mathrm{eV}, p=1.1$, во втором $Q\left(\tau_{\mathrm{eff}}\right)$ и $p(T)$ по [9].

На рис. 6 приведены распределения абсолютной величины скорости свободной конвекции расплава и тем- 

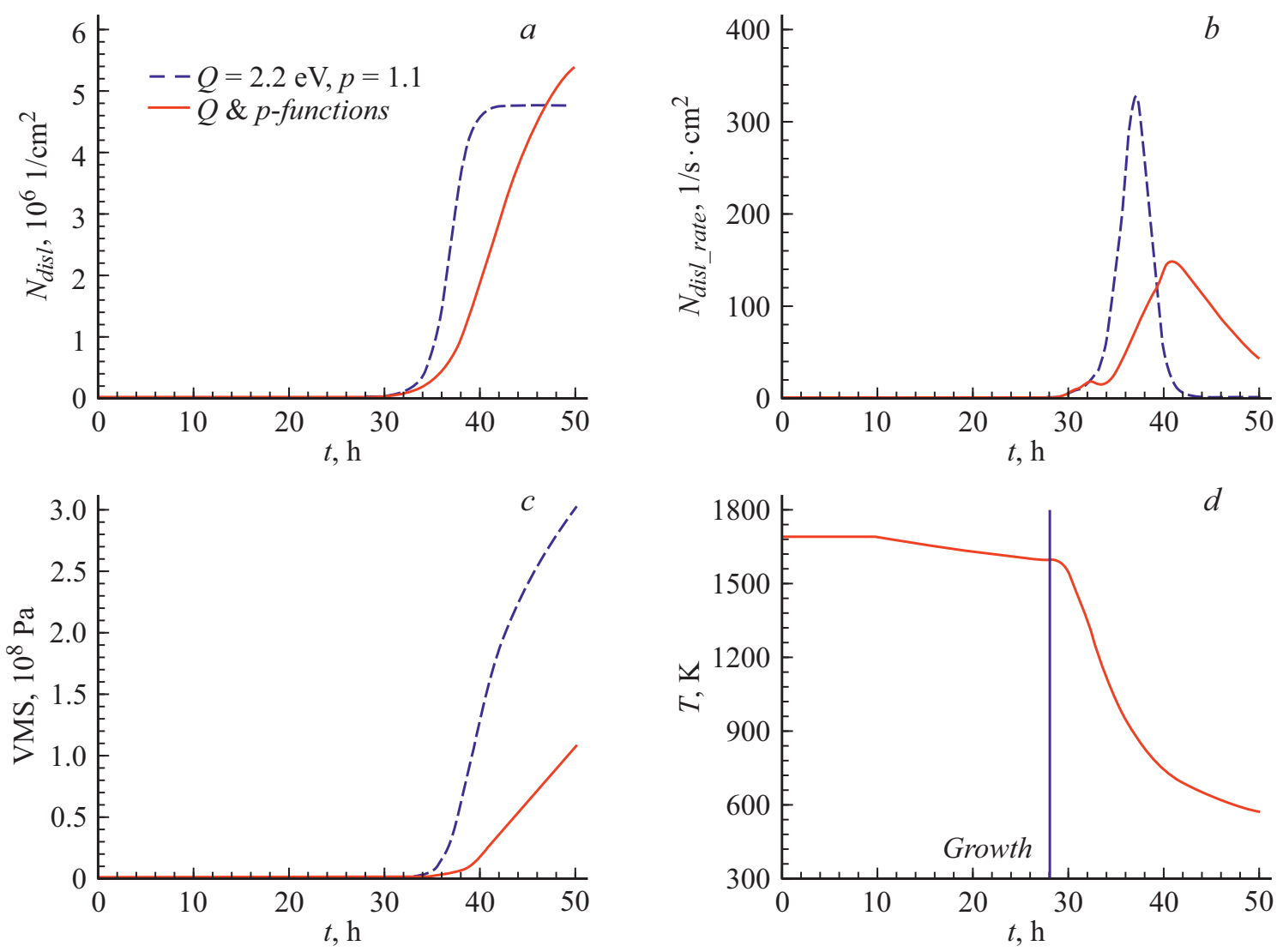

Рис. 8. Изменение параметров в течение процесса в точке $\mathrm{P}$ (рис. 7) при двух наборах $Q$ и $p: a-$ плотность дислокаций; $b-$ скорость роста дислокаций; $c-\mathrm{VMS}, d-$ температура.

пературы для седьмого часа процесса, $t=7 \mathrm{~h}$. Максимум скорости расплава $V_{\mathrm{abs}}=4.43 \mathrm{~mm} / \mathrm{s}$ формируется в нисходящем потоке около тигля. В структуре течения доминируют два вихря, расположенные один над другим. Максимум температуры локализован около периферийной части свободной поверхности расплава. Перегрев расплава по отношению к температуре плавления равен примерно $8 \mathrm{~K}$. Ростовой интерфейс кристалла достаточно ровный, с вогнутостью около боковой стенки тигля, обусловленный действием периферийного нисходящего течения.

На рис. 7 показано распределение в кристалле плотности дислокаций (рис. 7,a) и VMS (рис. 7,b) для момента завершения процесса, $t=50 \mathrm{~h}$. Если исключить относительно незначительную периферийную часть кристалла, которая в реальном производстве срезается при обработке, увидим, что при $Q=2.2 \mathrm{eV}, p=1.1$ максимум плотности дислокаций меньше, чем при зависимостях $Q\left(\tau_{\text {eff }}\right)$ и $p(T)$. В первом случае $N_{d i s l}=4.87 \mathrm{~cm}^{-2}$, во втором $N_{d i s l}=5.6 \mathrm{~cm}^{-2}$. Соответственно в первом случае плотность термических напряжений выше.

На рис. 8 показаны зависимости скорости роста кристалла, плотности дислокаций, напряжений и температуры в точке максимума $N_{d i s l}$ (отмеченной точкой в левой части рис. 7,a). Из них следует, что при $Q=2.2 \mathrm{eV}$, $p=1.1$ после $t=41 \mathrm{~h}$ величина $N_{d i s l}$ выходит на по- стоянный уровень (рис. $8, a)$. При использовании $Q\left(\tau_{\mathrm{eff}}\right)$ и $p(T)$ рост $N_{\text {disl }}$ продолжается до конца процесса охлаждения кристалла. Скорость роста дислокаций при фиксированных $Q, p$ достигает максимума при $t=37 \mathrm{~h}$ и практически полностью затухает при $t=41 \mathrm{~h}$ (рис. $8, b$ ). В расчете с $Q\left(\tau_{\text {eff }}\right)$ и $p(T)$ максимум скорости роста дислокаций сдвинут к $t=41 \mathrm{~h}$, и к концу процесса она лишь в 3 раза меньше абсолютного максимума. Значения VMS в той же точке кристалла $\mathrm{P}$ на рис. 7 при постоянных $Q, p$ выше, чем при $Q\left(\tau_{\text {eff }}\right), p(T)$. Разница между ними увеличивается с течением времени и в конце процесса трехкратна (рис. $8, c$ ).

Таким образом, использование $Q\left(\tau_{\mathrm{eff}}\right), p(T)$ в полном технологическом процессе меняет расчетную динамику VMS и $N_{\text {disl }}$ по сравнению с применением $Q=2.2 \mathrm{eV}$, $p=1.1$.

\section{Выводы}

Для повышения эффективности численного сопровождения технологических работ при моделировании DSS-процессов с тиглями квадратного сечения можно использовать приближенную $2 D$-геометрию печи, построенную по вертикальному сечению симметрии тигля, параллельному его боковой грани. Тестовые расчеты 
показали, что это приближение позволяет получить результаты, удовлетворительно согласующие с полным $3 D$-расчетом по температурным полям в большей части кристалла, а именно в объеме вертикального цилиндра, условно вписанного в $3 D$-тигель. Также наблюдается корреляция между $2 D$ - и $3 D$-результатами расчетов тепловых напряжений в приосевой части кристалла.

Для улучшения согласования $2 D$ расчетных мощностей нагревателей с экспериментальными рекомендуется использовать масштабный коэффициент $(4 / \pi)^{0.5}$ для радиуса $2 D$ модельного тигля, а также для соответствующих $2 D$-модельных нагревателей и теплоизоляции.

Сравнительные расчеты плотности дислокаций по модели Александера-Хаазена, выполненные с постоянными величинами параметров кристалла $Q, p$ и с эмпирическими зависимостями $Q\left(\tau_{\mathrm{eff}}\right), p(T)$, показали различную динамику роста $N_{d i s l}$, что необходимо учитывать при численной оптимизации полных технологических процессов получения кристаллов.

\section{Финансирование работы}

Работа выполнена при частичной финансовой поддержке Фонда содействия развитию малых форм предприятий в научно-технической сфере в рамках Международной программы ERA.Net RUS+, номер гранта $295 \Gamma \mathrm{P} / 21031$.

\section{Конфликт интересов}

Авторы заявляют, что у них нет конфликта интересов.

\section{Список литературы}

[1] Chen L., Dai B. // J. Cryst. Growth. 2012. Vol. 354. P. 86-92.

[2] Chen W., Zhou B., Ding J., Yu Y., Dong H., Zhong G., Huang X. // J. Mater. Sci. Eng. B. 2016. Vol. 6. P. 201-210.

[3] Wu Z., Zhongb G., Zhang Z., Zhou X., Wanga Z., Huang X. // J. Cryst. Growth. 2015. Vol. 426. P. 110-116.

[4] Zhou B., Chen W., Dong H., Zhou X., Qi F., Luo J., Huan X. // J. Mater. Sci. Eng. B. 2017. Vol. 7. P. 89-98.

[5] Электронный pecypc. Режим доступа: http://www.strsoft.com/products/CGSim/

[6] M'Hamdi M., Gouttebroze S., Fjaer H.G. // J. Cryst. Growth. 2011. Vol. 318. P. 269-274.

[7] M'Hamdi M., Gouttebroze S., Fjaer H.G. // J. Cryst. Growth. 2013. Vol. 362. P. 83-87.

[8] Alexander H., Haasen P. // Solid State Phys. 1969. Vol. 22. P. 27-158.

[9] Erofeev V.N., Nikitenko V.I. // Soviet Physics Jetp. 1971. Vol. 33. N 5. P. $963-966$.

[10] Nakano S., Chen X.J., Gao B., Kakimoto K. // J. Cryst. Growth. 2011. Vol. 318. P. 280-282.

[11] Smirnova O.V., Mamedov V.M., Kalaev V.V. // Cryst. Growth Des. 2014. N 14. P. 5532-5537.

[12] Wolfshtein M. // Int. J. Heat Mass Transfer. 1969. Vol. 12. N 3. P. $301-318$.

[13] Kuliev A.T., Durnev N.V., Kalaev V.V. // J. Cryst. Growth. 2007. Vol. 303. P. 236-240.
[14] Gao B., Nakano S., Kakimoto K. // J. Cryst. Growth. 2013. Vol. 369. P. 32-37.

[15] Maroudas D., Brown R.A. // J. Cryst. Growth. 1991. Vol. 108. P. 399-415. 